BULL. AUSTRAL. MATH. SOC.

VOL. 31 (1985), 83-88.

\title{
ON THE SPECTRUM OF THE BIHARMONIC OPERATOR IN A BOUNDED DOMAIN
}

\section{Pui-Fai Leung and Luen-Chau Li}

We obtain an asymptotically sharp lower bound for the sum of the first $k$ eigenvalues of the biharmonic operator.

Let $D$ be a bounded domain in $\mathbb{R}^{n}$ with piecewise smooth boundary $\partial D$ and consider the following two eigenvalue problems:
(I) $\left\{\begin{aligned} \Delta \phi+\lambda \phi & =0 \text { in } D ; \\ \phi & =0 \text { on } \partial D \text {; }\end{aligned}\right.$
(II) $\left\{\begin{array}{lll}\Delta^{2} \phi-\mu \phi=0 & \text { in } D, \\ \phi=\frac{\partial \phi}{\partial n}=0 & \text { on } & \partial D .\end{array}\right.$

We let

$$
\begin{aligned}
& 0<\lambda_{1}<\lambda_{2} \leq \lambda_{3} \cdots, \\
& 0<\mu_{1} \leq \mu_{2} \leq \mu_{3} \cdots
\end{aligned}
$$

denote the successive eigenvalues of (I) and (II) respectively. We have the well-known asymptotic formulas of Weyl [4]:

$$
\lambda_{k} \sim C_{n}(k / V)^{2 / n}
$$

Received August 1984. The authors are greatly indebted to the paper [2] of $\mathrm{Li}$ and Yau for much inspiration.

Copyright Clearance Centre, Inc. Serial-fee code: 0004-9727/85 $\$ A 2.00+0.00$. 


$$
\mu_{k} \sim c_{n}^{2}(k / v)^{4 / n}
$$

where $C_{n}=(2 \pi)^{2} B_{n}^{-2 / n}$ with $B_{n}$ the volume of the unit $n$-ball and $V$ the volume of $D$. In [3] (see also [2], p. 310) Polya proved that

$$
\lambda_{k} \geq C_{n}(k / V)^{2 / n}
$$

holds for $\mathbb{R}^{n}$-covering domains (that is, domains that tile $\mathbb{R}^{n}$ ) and conjectured this inequality for arbitrary domains.

Now it follows from the asymptotic formulas that

$$
\begin{aligned}
& \sum_{i=1}^{k} \lambda_{i} \sim \frac{n}{n+2} c_{n} V^{-2 / n_{k}(n+2) / n,} \\
& \sum_{i=1}^{k} \mu_{i} \sim \frac{n}{n+4} c_{n}^{2} V^{-4 / n_{k}}(n+4) / n,
\end{aligned}
$$

and recently $L i$ and Yau in [2] proved that

$$
\sum_{i=1}^{k} \lambda_{i} \geq \frac{n}{n+2} c_{n} V^{-2 / n_{k}}(n+2) / n \text {. }
$$

Since $k \lambda_{k} \geq \sum_{i=1}^{k} \lambda_{i}$ it follows that

$$
\lambda_{k} \geq \frac{n}{n+2} C_{n}(k / V)^{2 / n}
$$

For problem (I), we have the following well-known variational characterization ([1], p. 395),

$$
\lambda_{n}=\max _{\left\{v_{1}, \ldots, v_{n-1}\right\}} \min _{\substack{u v_{1}, \ldots, v_{n-1} \\ u=0 \text { on } \partial D}}-\int_{D} u \Delta u
$$

and on going through the proof in [1], p. 395, we can see that problem (II) can be similarly characterized by 


$$
\mu_{n}=\max _{\left\{v_{1}, \ldots, v_{n-1}\right\}} \min _{\substack{u \nu_{1}, \ldots, v_{n-1} \\ u=\partial u / \partial n=0 \text { on } \partial D \\ \int u^{2}=1}}(\Delta u)^{2}
$$

and hence

$$
\left.\mu_{n} \geq \underset{\left\{v_{1}, \ldots, v_{n-1}\right\}}{\max } \underset{\substack{u v_{1}, \ldots, v_{n-1} \\ u=0 \text { on } \partial D \\ \int u^{2}=1}}{ }\right\}(\Delta u)^{2}
$$

and so it follows from the Cauchy-Schwarz inequality that

$$
\mu_{k} \geq \lambda_{k}^{2}
$$

Therefore by applying Polya's theorem, we obtain

THEOREM 1. If $D$ is a $\mathbf{R}^{n}$-covering domain, then

$$
\mu_{k} \geq c_{n}^{2}(k / V)^{4 / n} \text {. }
$$

In view of these, it is natural to propose the following:

CONJECTURE. For any bounded domain in $\mathbb{R}^{n}$,

$$
\mu_{k} \geq c_{n}^{2}(k / V)^{4 / n}
$$

In this note we shall make a slight modification of the proof in [2] and show that:

THEOREM 2. For any bounded domain $D$ in $\mathbf{R}^{n}$, we have

$$
\sum_{i=1}^{k} \mu_{k} \geq \frac{n}{n+4} c_{n}^{2} V^{-4 / n_{k}}(n+4) / n \text {. }
$$

COROLLARY. $\mu_{k} \geq(n /(n+4)) c_{n}^{2}(k / V)^{4 / n}$.

Proof. This follows since $k \mu_{k} \geq \sum_{i=1}^{k} \mu_{i}$.

Proof of Theorem 2. We shall need the following extension of a lemma of Hormander ([2], p. 311). 
LEMMA 1. Let $f$ be a real-valued fronction defined on $\mathbb{R}^{n}$ such that $0 \leq f \leq M_{1}$ and let $m$ be a positive integer. Suppose

$$
\int_{\mathbf{R}^{n}}|z|^{m} f(z) d z \leq M_{2}
$$

then

$$
\int_{\mathbf{R}^{n}} f(z) d z \leq\left(\frac{n+m}{n}\right)^{n /(n+m)}\left(M_{1} B_{n}\right)^{m /(n+m)} M_{2}^{n /(n+m)} .
$$

Proof. Just replace 2 by $m$ in the proof in [2], p. 312, and everything goes through just the same.

Now let $\phi_{1}, \ldots, \phi_{k}$ be a set of orthonormal eigenfunctions corresponding to the eigenvalues $\mu_{1}, \ldots, \mu_{k}$ respectively and extend $\phi_{i}$ to be zero outside $D$. Following [2] we introduce the function

$$
\Phi(x, y)=\sum_{i=1}^{k} \phi_{i}(x) \phi_{i}(y)
$$

and consider its Fourier transform in $x$ which is given by

$$
\hat{\Phi}(z, y)=(2 \pi)^{-n / 2} \int_{\mathbf{R}^{n}} \Phi(x, y) e^{i x \cdot z} d x .
$$

We now let

$$
f(z)=\int_{D}|\hat{\Phi}(z, y)|^{2} d y
$$

Then from equations (6) and (4) of [2] we have

$$
0 \leq f(z) \leq(2 \pi)^{-n_{V}}
$$

and

$$
\int_{\mathbb{R}^{n}} f(z) d z=k
$$

As in [2] the crucial step now is to get an estimate of the left hand side of (*).

To do this we consider 


$$
\begin{aligned}
& \int_{\mathbb{R}^{n}}|z|^{4} f(z) d z \\
& =\left.\left.(2 \pi)^{-n} \int_{\mathbf{R}^{n}} \sum_{i=1}^{k}\left|\int_{D}\right| z\right|^{2} \phi_{i}(x) e^{i x \cdot z} d x\right|^{2} d z \\
& =\sum_{i=1}^{k}(2 \pi)^{-n} \int_{\mathbb{R}^{n}}\left|\int_{D} \sum_{j=1}^{n} z_{j}^{2} \phi_{i}(x) e^{i x \cdot z} d x\right|^{2} d z \\
& =\sum_{i=1}^{k}(2 \pi)^{-n} \int_{\mathbf{R}^{n}}\left|\int_{D} \phi_{i}(x) \Delta_{x} e^{i x \cdot z} d x\right|^{2} d z \\
& =\sum_{i=1}^{k} \int_{\mathbf{R}^{n}}\left|(2 \pi)^{-n / 2} \int_{D}\left(\Delta \phi_{i}(x)\right) e^{i x \cdot z} d x\right|^{2} d z \quad \text { (by Green's identity) } \\
& =\sum_{i=1}^{k} \int_{\mathbf{R}^{n}}\left|\Delta \hat{\phi}_{i}(z)\right|^{2} d z \\
& =\sum_{i=1}^{k} \int_{\mathbb{R}^{n}}\left(\Delta \phi_{i}(z)\right)^{2} d z \text { (by Parseval formula) } \\
& =\sum_{i=1}^{k} \int_{D} \phi_{i}(z) \Delta^{2} \phi_{i}(z) d z \\
& =\sum_{i=1}^{k} \mu_{i} \text {. }
\end{aligned}
$$

Finally, by applying Lemma 1 with $m=4, M_{1}=(2 \pi)^{-n_{V}}$,

$$
\begin{aligned}
& M_{2}=\sum_{i=1}^{k} \mu_{i}, \text { we obtain } \\
& \qquad k \leq\left(\frac{n+4}{n}\right)^{n /(n+4)}\left((2 \pi)^{-n} V B_{n}\right)^{4 /(n+4)}\left(\sum_{i=1}^{k} \mu_{i}\right)^{n /(n+4)}
\end{aligned}
$$

which simplifies to

$$
\sum_{i=1}^{k} \mu_{i} \geq \frac{n}{n+4} c_{n}^{2} V^{-4 / n_{k}(n+4) / n} .
$$

ADDED REMARK. After we had completed this paper we were informed by Professor S.T. Yau that Professor M.H. Protter has obtained similar results 
independently.

\section{References}

[1] P.R. Garabedian, Partial differential equations (John Wiley \& Sons, London, New York, 1964).

[2] P. Li and S.T. Yau, "On the Schrodinger equation and the eigenvalue problem", Comm. Math. Phys. 88 (1983), 309-318.

[3] G. Polya, "On the eigenvalues of vibrating membranes", Proc. London Math. Soc. (3) 11 (1961), 419-433.

[4] H. Weyl, "Das asymptotische Verteilungsgesetz der Eigenwerte linear partieller Differentialgleichungen (mit einer Anwendung auf die Theorie der Hohl raumstrahlung)", Math. Ann. 71 (1912), 441-479.

Department of Mathematics,

National University of Singapore,

Kent Ri dge,

Singapore 0511 . 